\title{
May the Plan be with you! \\ A Usability Study of the Stimulated Planning Game Element Embedded in a MOOC Platform
}

\author{
Alessandra Antonaci ${ }^{1}$, Roland Klemke ${ }^{1}$, Kim Dirkx ${ }^{1}$, Marcus Specht ${ }^{1}$ \\ ${ }^{I}$ Welten Institute, Open University of the Netherlands, Heerlen, Netherlands, \\ \{alessandra.antonaci, roland.klemke, kim.dirkx, ,marcus.specht\}@ou.nl
}

\section{Abstract}

Goal achievement is a measure of success; this could be particularly true in Massive Online Open Courses (MOOC), which are approached by a massive audience with an enormous variety of needs. Despite the huge number of MOOC users, it is unlikely to find solutions that allow them to pursue their individual goals. To overcome this issue, we have developed a first prototype of the Stimulated Planning (SP) game element, inspired by the implementation intention theory and by our experience with strategy games. In this study, the $S P$ prototype is presented and its performance assessed via a new combination of three methodologies: usability test, eye tracking and the retrospective thinkaloud technique. The results are promising and contribute to the field of gamification of MOOC at three levels: at conceptual level, by introducing and interpreting a new theory for gamification design; at design level, by demonstrating that is possible to design advanced gamification for MOOCs; and at assessment level, by applying a new methodology for MOOC gamification design assessment.

Keywords: Game Element, Gamification Design, Usability, Eye-Tracking, Think-Aloud, Implementation Intention, Stimulated Planning;

\section{Introduction}

Massive Online Open Courses (MOOCs) are available for a variety of domains and are mostly accessible for free. While these courses generally attract a big number of users, they record incredibly high dropout rates, meaning that there is a huge difference between the number of users who enroll in the courses, and those who actually reach the end. One of the reasons for user' abandonment lies in the lack of personalization that often characterizes these courses MOOCs generally offer the same package of content and materials to all users, no matter what their motivations, intentions, pre-knowledge, educational/cultural background and skills are. Furthermore, the majority of MOOC providers do not support personalized feedback functionalities that could enable course designers to actually build an adaptive course for heterogeneous user groups. As a consequence, MOOC learners are unable to follow a personal path, and do not have the chance to fulfil their own intentions and reach their personal goals within the course.

Intentions and goal achievement are the focus of the implementation intention theory [2], which was developed and applied in health psychology. Despite its empirical validity, this theory has not been implemented in MOOCs yet. According to this theory, people who specify their goals with a more concrete implementation intention (plan), have a higher chance of achieving their goals compared to people who only have vaguely specified goals [3]. 
According to Gollwitzer [1], to raise the probability of a goal achievement with an implementation intention the following steps need to be taken:

1. identify a goal;

2. specify a (1) if-then plan;

3. and a (2) coping plan.

To detail (1), if-then plan, an if and then component need to be specified. Hence, describing "the if-component of an implementation intention" [4] implies specifying "when and where one wants to act on this goal" [3], while the then-component requires detailing the how the if will be implemented. As far as the (2) coping plan is concerned, its specification requires picturing the inconveniences that could undermine the if-then plan and the strategies to be performed in case these may occur [5], [6].

In literature, several studies investigate users' intentions within MOOCs [7]-[9], defining and reporting on the general state-of-the art and contributing on a theoretical level to the field. In this article, we aim to contribute on a practical level, by introducing a solution that will enable users to fulfil their goals within a MOOC. Our solution is an online tool, which consists of a game design element aiming at facilitating MOOC learners in creating and implementing their own path defining if- then and coping plans.

The game element we refer to is called Stimulated Planning (SP). The SP as game design pattern is described in [10], it is generally used in strategy games which "give players a sense of Empowerment and is the effect of Predictable Consequences and either Freedom of Choice or an Illusion of Influence. The activity of planning also gives Cognitive Immersion but may cause Analysis Paralysis if the Right Level of Complexity is not achieved" [10]. In other words, by giving users the chance to plan their strategy they feel empowered, furthermore this planning activity may help them to concentrate better. However, in designing the SP the difficulty level has to be kept in mind, since a task that is too hard could block the user, while one that is too easy will demotivate or bore him.

We designed the SP component for MOOCs, taking inspiration from the strategy games and the implementation intention theory, giving MOOC users the chance to personalize their own path by detailing their if- then and coping plans in relation to the course.

Before embedding the SP component on a large scale, in a MOOC environment, the assessment of this innovative game element for MOOCs is needed. The assessment consists of a formative study, conducted using an innovative methodology, presented in this paper, which focuses on the SP usability and investigates the ways in which it can be improved. Referring to International Organization of Standardization (ISO) the three attributes mentioned above, they are defined as follows: "effectiveness" refers to the users' ability to achieve their goal by using the system; while "efficiency", moves the attention to the recourses users consume in order to achieve their goal/s. Last, but not least, the "satisfaction", refers to users' perception, feeling, while using the system [10]. According ISO a software product "is usable when it allows the user to execute his task effectively, efficiently and with satisfaction in the specified context of use" [11]. Recently another characteristic has been introduced to measure usability: "learnability", which determines the time users need to learn how to use the interface or system [11]. Hence, each attribute of usability focuses on different aspect of the system: goal achievement (effectiveness); use of resources (efficiency); orientation (learnability) and satisfaction, all equally important to determine the usability of a system.

These attributes are generally evaluated via the System Usability Scale (SUS) questionnaire. However, to assess the usability of a system and more specifically the interaction with the user interface, a widely applied technology is eye tracking. It determines users' visual attention patterns [12], based on which it is possible to derive usability considerations. Lately, eye tracking (ET) has been increasingly used in Technology Enhanced Learning (TEL) to evaluate and consequentially enhance the instructional design of computer-based environments [13]. While users perform specific tasks, such as reading, scanning an image, or entering data, the eye tracker captures the eye 
movements, more specifically, the fixations (a pause of the eye movement on a specific area of the visual field), and saccades (rapid movements between fixations) [14].

Eye movements alone cannot give us enough information to improve the user experience of our game element. As a matter of fact, not all eye movements are voluntary. Besides, the interpretation of quantitative information, i.e. the duration of a fixation, could be done in two different and opposite ways: it may mean that the user has found that specific area/object particularly interesting but it may also mean that s/he found that area (or object) particularly difficult to interpret, thus s/he needed to look at it for a longer period of time. Therefore, along with eye tracking it is necessary to collect qualitative data, coming, for instance, from the users' voice and experience. These data can enable researchers to better understand the strengths and the weaknesses of the system and can be gained via the thinkaloud (TA) method. There are two types of TA: concurrent think-aloud (CTA) which requires users to verbalize their thoughts while performing the task/s required in that specific interface (and or system), and retrospective think-aloud (RTA) which implies users to recall their operational process only after having performed the task/s. Although both methods can provide interesting insights, they also have their own drawbacks: CTA, "influences the user's actual operation"; while with RTA "when recalling, the user will add or delete some information, which decreases the data accuracy"[12].

In the framework of this study, based also on Yang's [12] analysis, we chose to perform a usability test (SUS), eye tracking study and RTA, this last was preferred to the CTA because it could overcome the data accuracy issue by combining it with the two other methods (SUS and ET). Furthermore RTA has proven to be "more efficient for analysing the cognitive factors of usability issues in-depth"[12] and combined with the other methods it will enable us to answer the following research questions (RQ) that investigate three different levels:

$1^{\text {st }}$ Overall usability evaluation level - RQ1- How usable is the stimulated planning (SP) tool?

Sub question (SQ) 1- To which extent is the SP tool efficient, effective, learnable and satisfactory for users? (Methods used: SUS, ET and RTA)

$2^{\text {nd }} \mathrm{HCI}$ interfaces evaluation level - RQ2- On each interface of the SP, do users focus on the areas considered relevant by the designers? (Method used: ET)

$3^{\text {rd }}$ Improvements level - RQ3- Which dimensions (navigation, layout, data entry, terminology used) received more requests for improvement by users? (Method used: RTA)

Based on the data collected, in the conclusion section we have drawn guidelines for gamification designers on how to design and implement a web-based application that can enable MOOC users to plan and achieve their own goal within a massive, online, open course, having an impact on MOOC completion rate (the authors consider completion rate as relative to users' intention and interest [15]). Furthermore, an innovative way to study the usability of gamified solutions for MOOC is introduced: combining SUS, eye tracking and thinking aloud measures, it enables researchers to collect rich data to assess usability and design issues in a more elaborate and objective way.

The remainder of the paper is organized as follows: after presenting related work and the conceptual and technical characteristics of the SP tool, the usability study is detailed. Then the data collected are discussed and finally our conclusions and future work are outlined.

\section{Usability studies in the field of gamification of MOOCs}

Despite the availability of studies on the usability of MOOCs [16], [17], the literature is lacking usability studies in the field of gamification of MOOCs. One of the reasons can lie in the fact that there are relatively limited numbers of gamified MOOCs available. As a 
pag. 52

matter of fact, gamification as a phenomenon dates back to 2012 [18] which is also the year in which MOOCs flourished [19]. Consequently, the first examples of gamified MOOCs can be found from 2014 onwards, since then, the game elements most implemented have been PBL (Points, Badges and Leader boards), which do not require in-depth usability studies due to their simplicity in design.

However, by digging into the literature, we found two examples of usability studies of game elements applied in MOOCs: [20] and [21].

In these two examples, methods like the eye tracking and the RTA are not applied. More specifically, [20] presents, among others, the results of a usability study of their application, performed by means of: SUS (system usability scale) and UEQ (user evaluation questionnaire). While [21], uses only an assessment tool, the Nielsen questionnaire, composed of 51 Likert-scale items [21].

In the first case, the system evaluated was "the training system (combination of smartphone app and activity-tracking device)" [20], which consisted of a smartphone app, developed within the "MOOC project" [20]. While in the second case the authors tested their gamified system which was designed to enhance student collaboration within a SPOC (Small Private Online Courses) [21].

In our case we aim to contribute to the field by performing a usability study, focusing on evaluating our SP embedded in the real scenario of application: a MOOC platform, more specifically Open edX.

\section{Stimulated Planning for MOOC: our prototype}

\subsection{The concept behind the SP prototype}

The concept behind the Stimulated Planning (SP) has been described in our previous work [22], and it takes inspiration from (1) the implementation intention theory [23] and (2) the strategy games, such as real time strategy (RTS) games, which require players to plan their action and tactic too (i.e. in the market can be found "Dune II: The Building of a Dynasty", which founded the genre in 1992).

In a first step, we designed the structure of our game element based on the SP design pattern from [10]. Then, we embedded the game mechanisms and principles, taking into consideration that, to encourage users' empowerment and illusion of influence, the SP game element needed to be designed with the right level of complexity.

As stated above, the implementation of an intention starts with a "goal-relevant situational cue". In our MOOC application scenario, the cue consists in learning the topic presented in the course. Furthermore, it has to be underlined that each MOOC user decides independently and is free to enrol in a course. They can choose among several types of MOOCs, providers and disciplines. Therefore, being in a MOOC can be already considered as a "goal- relevant situation"/cue for a MOOC user. According to [3], once the cue is defined, performing implementation intention requires the subject to link the cue to "an instrumental goal-directed response" [3], which is the if-then plan.

To enable users detailing an if-then plan via the SP, they need information to create a mental representation of the possible options and obstacles related to the cue (the course). Therefore, we decided to present the course outline by means of a video. Afterwards, we asked them to choose from the following three options: following (1) all the course or (2) some selected lessons/activities, and (3) browsing around.

To enable users' awareness and to facilitate the planning, we provided them with information about the duration of each lesson/activity, also asking them how much time they are willing to spend on the course. Based on the data gathered, the SP elaborates a feedback which informs users on the average time they will need to complete the action/s selected. 
With this awareness, users are invited to plan their lesson/s (or the full course) by dragging and dropping them from a menu into a calendar, defining the if (what and when) of their if-then plan. The calendar enables then the definition of the if component, and the system gives users the option to decide how to follow their lesson: in freedom and autonomy.

According to implementation intention theory, for executing the implementation of an intention, users are required to define a "coping plan", which implies, in principle, to gain awareness of possible inconveniences that could undermine the achievement of the designated goal (in our case, following the entire course or part of it), and also to find the possible solutions. Following the format: "I intend to do $\mathrm{Y}$ when situation $\mathrm{Z}$ is encountered" [3]. To enable users to define their coping plan of the implementation intention, we asked them to write down or choose among given examples, the possible obstacles and solutions that could occur in a MOOC- based learning scenario.

To boost users' empowerment and illusion of influence, the SP gives users the chance to choose among few options within the course, giving the idea and perception that they can actually do what they like in the course, without following a pre-defined structure. Furthermore, each user is provided with a visual representation of his/her progress and s/he can decide to re-plan or change it, having an influence on their action and planning activities.

\subsection{The process layer}

To provide a better understanding on how the SP works within the Open edX platform, the process level needs to be specified. The game element is not visible to all the users that enrol in the course. The users are randomly divided in control and treatment groups, (see figure 1), and the six widgets of SP are visible to users in the treatment group.

Widget is a generic term for the part of a GUI (Graphical User Interface) that allows the user to interface with the application and operating system"[35]. In the framework of this paper, the following terms are used: interface, page and widget. The first two: interface and page refer to visual design, while the term widget is used to refer to the graphic component and its controlling program.

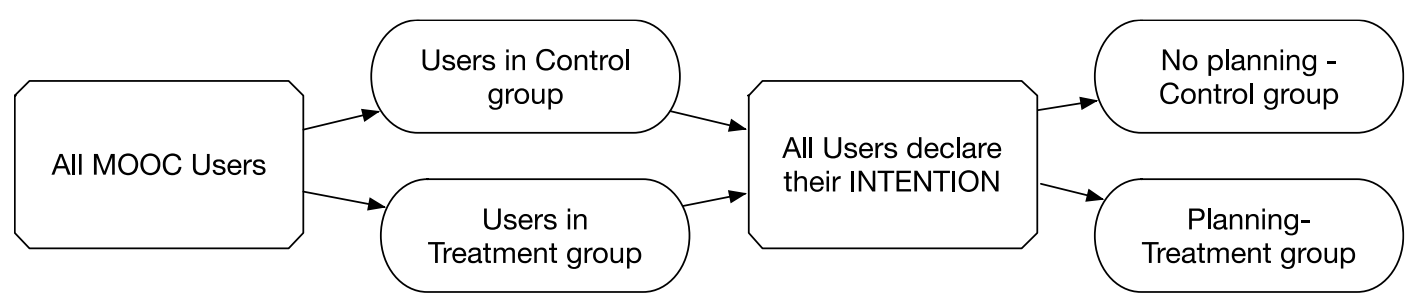

Figure 1. MOOC users' subdivision in control and treatment groups

At the beginning of the course, all users are asked to declare their intention within the course by choosing among the three options given (following (1) all the course or (2) some selected lessons/activities, and (3) browsing around). Afterwards, the SP is visualized by users in the treatment group.

To transfer the game element into practical applicability within the platform, we firstly designed an interaction process, of how the standard use of a MOOC environment would be enhanced, when SP is available. Within this process, we include steps to enable users' implementation intention. Figure 2 shows the overall process comprising (1) the general steps of the MOOC process (registration and enrolment, course start, and learning activities), for all users, (2) the steps for implementation intention (defining if- then and coping plans), and (3) the steps for stimulating the planning (planning support, progress tracking, feedback visualization, and notification for planning). 


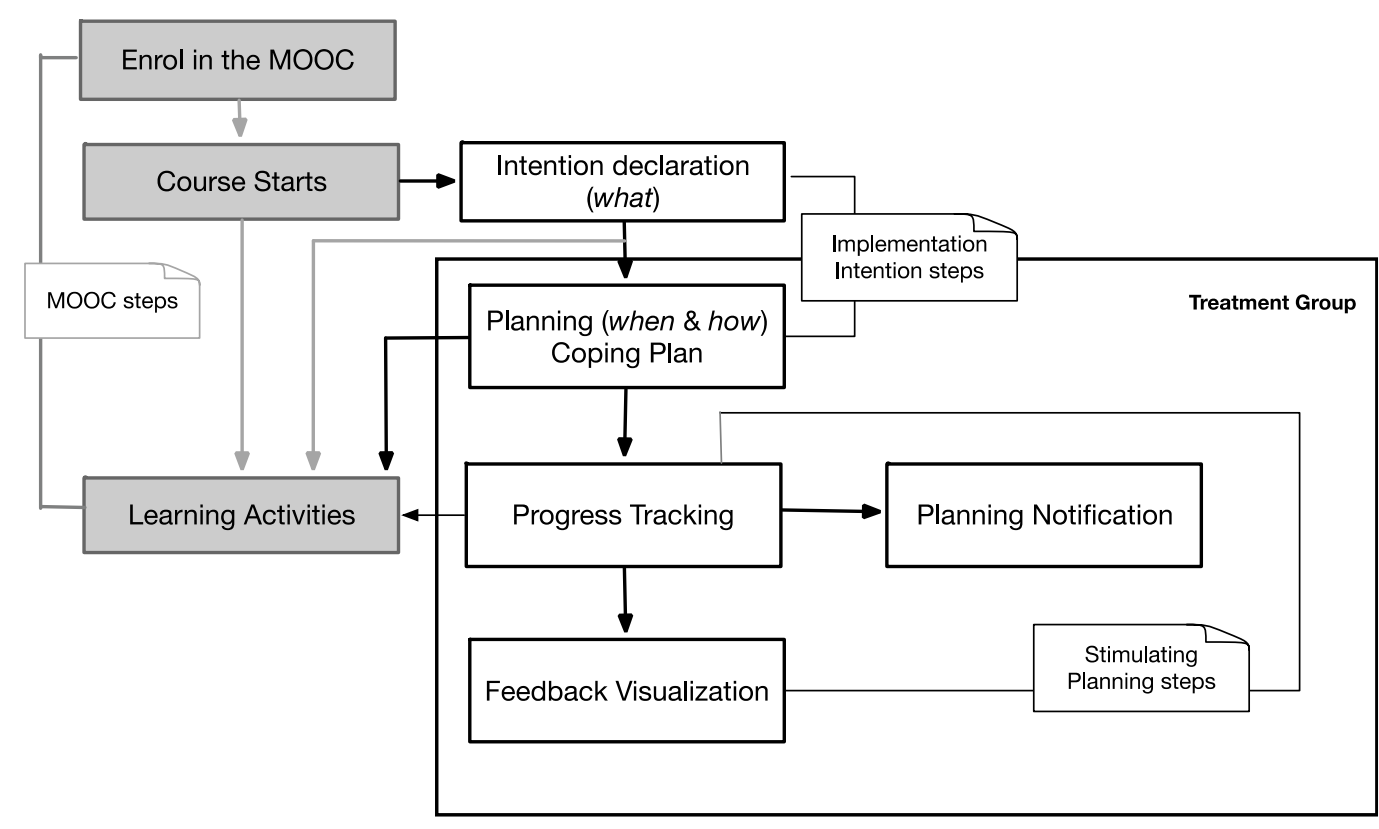

Figure 2. Process through MOOC (grey) and Stimulated Planning Application (white)

The steps belonging to MOOC activities will not be further described in this article, as they are not part of our experiment design but cover aspects such as login, enrolment, course access, and Open edX course structure and layout. The steps related to the implementation intention imply users to: declare their intention $\left(1^{\text {st }}\right.$ widget $)$ with respect to the course. They are asked to specify if they intend to complete the whole course, only selected topics or if they just intend to browse the course. By this, users define what they would like to follow. In the $2^{\text {nd }}$ widget we ask users about the available time per week and provide them with info ( $3^{\text {rd }}$ widget) about their selection, the total amount of hours, and an estimation of the time needed to complete their activities. Based on this information, users can plan ( ${ }^{\text {th }}$ widget) via an integrated calendar (defining when and how). After this, users are asked to detail the coping plan, by thinking about possible inconveniences that could undermine the just made plan, and which solutions they could find to solve these problems ( $5^{\text {th }}$ widget $)$.

The Steps for stimulating the planning consists of four features: (1) planning support, within the $3^{\text {rd }}$ widget and at any time during the course runtime users can revisit their plan and adjust it to their needs (re-plan); (2) The progress tracking step that hooks into the learning activities and tracks progress according to the plan. The data gathered this way is used to give immediate feedback to the learner (3-feedback visualization) and to send regular reminder messages to them too (4-notification for planning). Feedback and notifications inform the learner about progress achieved with respect to the plan (completed items), about upcoming planned activities, about items, which the learner is late with according to the plan, and about activities not yet planned.

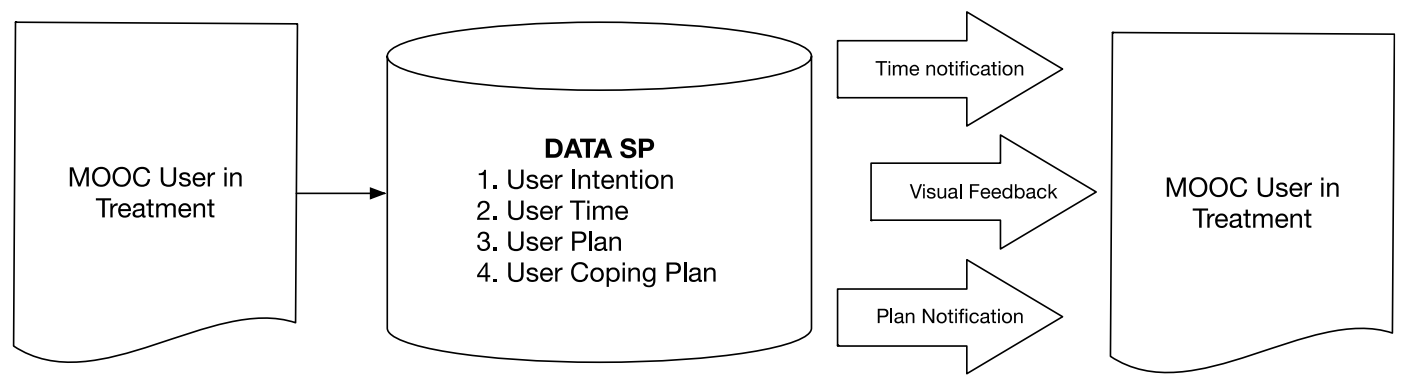

Figure 3. Data gathered by the SP from UX and feedback given to them by the $S P$ 
Figure 3 shows how the users in the treatment group, who visualize our SP, generate data that the SP captures: (1) specification of their intention; (2) their time availability; (3) plan data; (4) and coping plan data. Then the users receive back (1) time notifications in the Memo Page, which inform them about the time needed to select the actions in which they are interested; (2) visual feedback with their progress and (3) plan notifications to stimulate the planning

\subsection{Architecture layer}

The above detailed processes (implementation intention and stimulated planning steps) are realized in a separate web-based application independent from Open edX, which is connected to it through user interface level widgets, which are integrated into the course content. A key reason to implement a separate application lies in designing the SP independently from the specific platform (Open edX) but also, to make it suitable for other MOOC providers. Furthermore, this architecture allows us to keep user-related personal data (maintained by the MOOC platform) strictly separate from the data gathered for our stimulated planning application, which simplifies anonymization of experimental data.

The SP is implemented using a three-tier architecture with database backend, application server, and web-based front-end widgets designed for integration in a MOOC platform. Figure 4 represents the core system architecture of the SP application, where the white elements symbolise the newly implemented stimulated planning application and the grey elements represent the previously existing elements of the MOOC platform (which will not be further described).

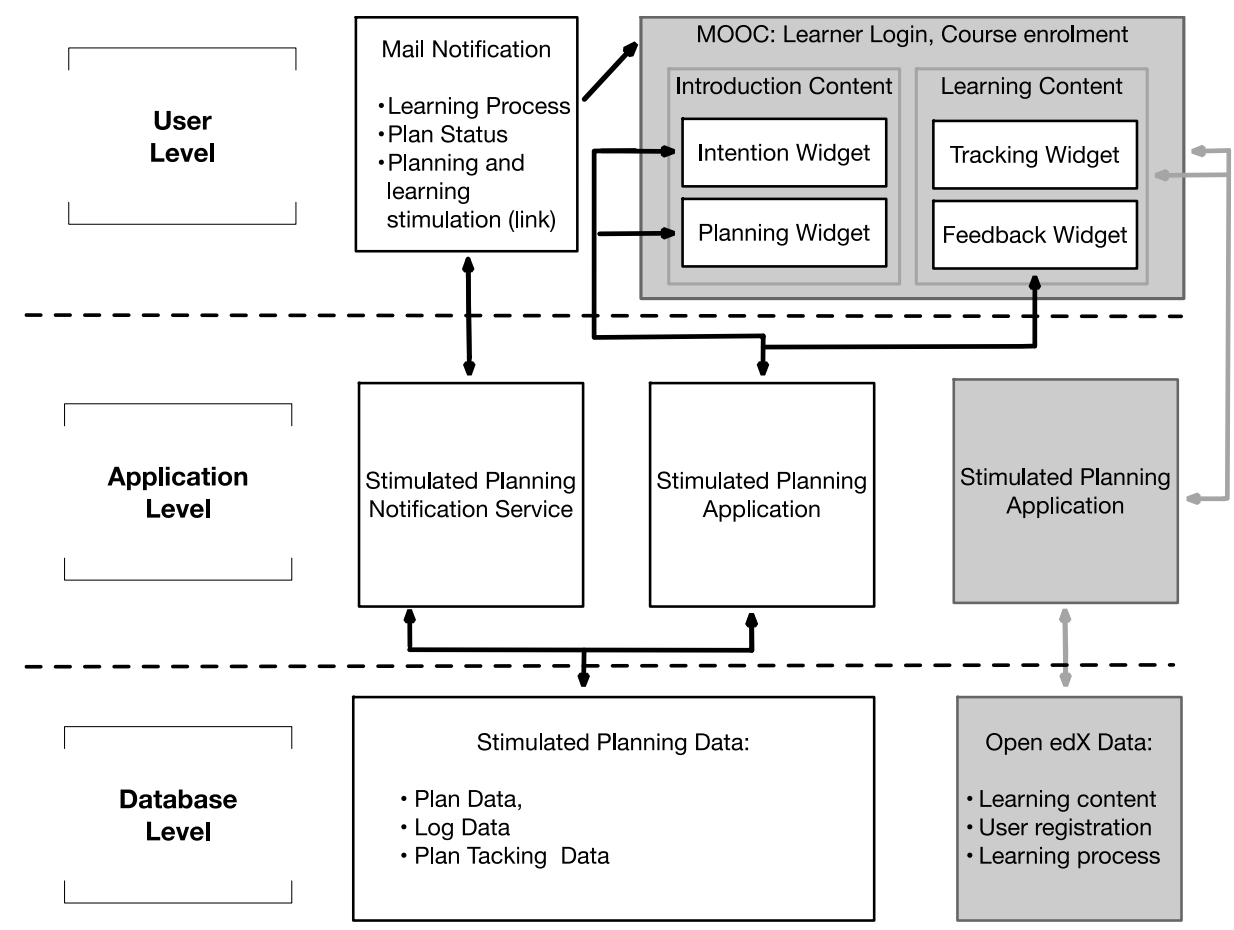

Figure 4. General architecture of the stimulated planning application (white) and its integration into the Open edX MOOC environment (grey)

- Database level: The database backend stores user plans, log data with respect to both planning activities and learning activities, as well as the corresponding progress tracking data (i.e. the data analysing, in how far a user stays within the plan, is ahead of the plan, or is late with respect to the plan).

Application level: the application server reads the data stored on the previous level to feed the user interface level widgets accordingly (intention widget, planning widget, progress widget). The application server is responsible for the personalisation of the user 
interface to the corresponding user intention, plan-related data, and progress feedback. The application level also comprises the notification service. According to a schedule, this service sends out personal notification mail messages to enrolled users informing them about their plan related progress and to remind them of upcoming or missed activities.

User level: the web-based front-end widgets of the SP application are responsible for visualising all intention- and plan-related data to the learner and for receiving all SPrelated user input (intention declaration, planning, re-planning, progress tracking). These widgets are integrated into the overall user interface of the MOOC platform in such a manner, that they appear as one system.

While the MOOC platform handles learner login, course registration, course navigation, and content access (as described in section 3.2), all interactions with the SP application are offered to the user through the front-end widgets, which are embedded into the course activity pages. The set of widgets comprises: the intention widget, which corresponds to the intention declaration process step; the planning widget, which corresponds to the planning process steps involving the calendar and the coping plan; the tracking widget, which is an invisible element that keeps track of the learner progress in regard to the intention and plan; and the feedback widget, which informs the learner about intention- and plan-related progress and which allows the learner to start the re-planning.

\section{Methods and data collection}

Following ISO 9241-11, which states that usability cannot be defined without the context of use, we tested the usability of our game element SP while inserted in the MOOC Open edX platform, on which our course was deployed. Consequently, in order to answer the RQs (1-3) we have designed this usability study in three steps, applying three different but complementary methods: (1) usability test, using the system usability scale (SUS) [24]; (2) Eye tracking (ET) and (3) Retrospective think-aloud (RTA) interviews.

The first step, via SUS, provides insights on the overall usability and on its attributes: effectiveness, efficiency, users' satisfaction and learnability of the SP (RQ1, SQ1). While the second step, via ET (2), enable us to gain information on the HCI, by reconstructing the visual pattern users perform on a page, and on specific part of the interface (the area of interest- AOI) (RQ2). The SP is composed of six different interfaces, as described in paragraph 3. Lastly the third step, with the RTA (3), enables a deeper understanding of the eye movement data and allows us to gather important suggestions on how to improve the usability of the SP (RQ3).

\subsection{Measures}

Via the means of SUS, the measurement of the following usability attributes [25] has been performed: efficiency, effectiveness, users' satisfaction and learnability of the SP. We adapted the SUS [24] with the modifications suggested by [25] as a 10 items questionnaire with a five points Likert scale (1-totally disagree, 5-totally agree). More specifically in the SUS, the items particularly dedicated to learnability are the two and four, (see Table 1) while for calculating all the others attributes, all other values need to be taken into consideration, (except two and four).

Regarding the ET, the data have been measured on two levels:

1) page level: for each of the six different interfaces of the SP we first calculated the Average Fixation Duration and blink frequency;

2) Areas of Interest (AOIs) level: we zoomed in on specific parts of each of the six interfaces of the SP. The following AOIs have been identified: Open edX layout; instructions given; the task area and the navigation (SP buttons), see Figure 5. In terms of measure, on each interface and AOI, we measured the Normalized Dwell average, the revisits average and the fixation duration average to gain insight on the viewing behaviour 
within these determined areas of each interface. By using these AOIs we can determine if the users are distracted or not from the Open edX layout; if the task area receives the main attention and focus, as it should; if the instructions are read or not, and last but not least if the participants use the SP buttons to proceed or the Open edX ones. More specifically about these measures:

- Average Fixation Duration is "the sum of fixation times divided by number of fixations" within the page [26] and gives us information on average time users spent looking at the interface or at a specific area of the page.

- blink frequency is the count per second of the blink that the eye of each participant produces, this measure is related to the cognitive load the participant is exposed to.

- Normalized Dwell average, is the average value of the sum of all the visits and the duration of all the saccade and fixations within the AOI. This average value is normalized in accordance to the size of the stimulus (i.e. the interface of the SP) and the AOI size (coverage). This value can give us hints on the reading and exploration patterns performed by the eye of our participants in specific areas of the widgets of our game element.

- Revisits average, is the mean value of the sum of the revisits count on a specific AOI.

\subsection{Equipment}

To perform the ET, we used the $250 \mathrm{~Hz}$ RED remote desktop eye tracking system from SMI Ltd. and the microphone of an external camera to record the comments of users while performing the RTA.

\subsection{The participants}

To determine the sample size in usability studies, there are specific numbers ( 5 and 30 ) to which the main literature refers to. This is mainly because [27]-[29] state that 5 participants can "identify (virtually) all of the" usability problems [30]; while Pernice and Nielsen "recommended the number 30 for eye tracking study" and stable heat maps [31]. Both these studies have limitations, as explained in [31]. Based on considerations exposed in [31], and the model developed by [32], we concluded that the sample size for our usability study, via SUS, and via ET and RTA, should be 17 test users, divided in two different groups.

The first group, composed of 8 users, was involved in the first step of the study (SUS). Participants had an average age of 30 years; the majority were men (5/8) with a computer science background (6/8) and none of them had used the SP prototype before.

While the second consisted of 9 participants, who took part in the ET and RTA studies, with an average age of 31 years; the majority were women (6/9) and belonged to different backgrounds (psychology, learning science, web-design, computer science). None of them had used the SP prototype before.

\subsection{Procedure}

The 8 candidates of the SUS study were invited via email to enrol in the MOOC, on which the SP was deployed, and after completing the essay required in the course, which implied the use of the SP, they were asked to assess its usability. This entire first step of our study took place online.

While as far as the setting up of the ET and RTA is concerned, it was in presence. After being invited to the study, participants were introduced to the ET techniques, informed on the procedure and the data collection and its storage. Subsequently, after the participants consent, the experiment started. At first, participants were introduced to the context of the study: a MOOC on computer security composed of four modules. Second, they were invited to "choose and declare what topic you want to focus on, according to your own preferences". Third, their eye movements were recorded while using the SP. 
pag. 58

After having used the SP and followed the activities in the MOOC, each participant was invited to narrate retrospectively his/her experience with the SP, by watching the ET video of their performance. For each SP interface, participants have been asked to comment on the following dimensions: layout; terminology/comprehension; data entry; feedback; navigation (SP buttons) and of any suggestions to improve the usability of the SP.

\section{Results}

\subsection{Overall usability evaluation level (SUS)}

The overall SUS score for the SP prototype tested revealed a mean score of 75.94, which is considered a score above average. As far as learnability of the SP is concerned, (looking at items two and four) SP yields a mean score of 73.75, while for the perceived efficiency, effectiveness, users' satisfaction the mean value is 76.67 .

Table 1 reports the questions and the mean values per each questionnaire item. Based on these data it is possible to gain more insights into distribution of the scores given for each value.

Table 1. SUS results per item

\begin{tabular}{c|l|c}
\hline No. & Items & M (SD) \\
\hline 1 & I think that I would like to use the system frequently & $3.63(0.74)$ \\
\hline 2 & I found the system unnecessarily complex & $4.25(0.89)$ \\
\hline 3 & I thought the system was easy to use & $4.25(0.89)$ \\
\hline 4 & I think that I could use the system without the support of a technical person & $4.63(0.74)$ \\
\hline 5 & I found the various functions in the system were well integrated & $3.75(1.28)$ \\
\hline 6 & I thought there was a lot of inconsistency in the system & $1.88(0.99)$ \\
\hline 7 & I would imagine that most people would learn to use the system very quickly & $4.50(0.53)$ \\
\hline 8 & I found the system very awkward to use & $1.75(0.89)$ \\
\hline 9 & I felt very confident using the system & $4.25(0.89)$ \\
\hline 10 & I needed to learn a lot of things before I could get going with this system & $1.75(1.16)$ \\
\hline
\end{tabular}

\subsection{HCI level (eye tracking)}

\section{HCI- Data on page level}

\begin{tabular}{c|l|c|c}
\multicolumn{3}{c}{ Table 2.Eye tracking Data } \\
\hline No. & \multicolumn{1}{|c}{ Pages } & $\begin{array}{c}\text { Fixation Duration } \\
\text { Average }[\mathbf{m s}] \\
\boldsymbol{M}(\boldsymbol{S D})\end{array}$ & $\begin{array}{c}\text { Blink Frequency } \\
\text { [count/s] } \\
\boldsymbol{M}(\mathbf{S D})\end{array}$ \\
\hline 1 & Intention Page & $296.73(26.54)$ & $0.15(0.12)$ \\
\hline 2 & Time Page & $353.96(75.15)$ & $0.09(0.11)$ \\
\hline 3 & Memo Page & $329.44(48.98)$ & $0.13(0.12)$ \\
\hline 4 & Plan Page & $369.15(37.15)$ & $0.11(0.08)$ \\
\hline 5 & Plan "b" Page & $378.29(83.22)$ & $0.20(0.12)$ \\
\hline 6 & End Page & $282.19(58.27)$ & $0.04(011)$ \\
\hline
\end{tabular}

Table 2 displays the fixation duration average and blink frequency values for each of the six interfaces of the SP.

The fixation duration average values highlight that participants spent most time reading the "plan b page" $(M=378.29 ; S D=83.22)$ followed by the "Plan Page" $(M=369.15 ; S D$ $=37.15$. $)$ and the "Time Page" $(M=353.96 ; S D=75.15)$. 
Furthermore the "plan b page" recorded the higher blink frequency $(0.20 / \mathrm{s})$, followed by "the intention page" $(0.15 / \mathrm{s})$ and the "memo page" $(0.13 / \mathrm{s})$. The "end page" has the lowest number of blinks ( $0.4 / \mathrm{s})$ (see Table 2$)$.

First explanation to these data can be due to the fact that the "plan $b$ page" is the interface with more text, as a logical consequence, participants needed more time to read the instructions (so high fixation duration average). In line with our expectations the high cognitive load (therefore blinking) is registered on the first interface of the SP, because users needed to get acquainted with the SP design (more details can be found in the discussion session).

\subsection{HCI-Data on AOI}

The following figure (Figure 5) represents the first interface of the SP, it is the page in which users declare their intentions in relation to the course. As visible from the figure the layout of the interface follows this logic: both the very top and the very bottom of each interface of the SP are busy with the Open edX dashboard and navigation buttons (these cannot be modified), immediately below the Open edX dashboard the instructions we designed are presented, then the middle of the page is dedicated to the task that the users have to perform and the bottom presents the navigation buttons we designed.

Considering that each interface of the SP is designed using the same logic in the layout, it was possible to draw the same AOI in each page and compare them to investigate the RQ2 and understand if there were flaws in the design.

We have named the AOI as follows: Open edX Layout (on top); AOI- instructions (immediately below the dashboard); AOI- task area (middle) and AOI- SP buttons ( below) (Figure 5).

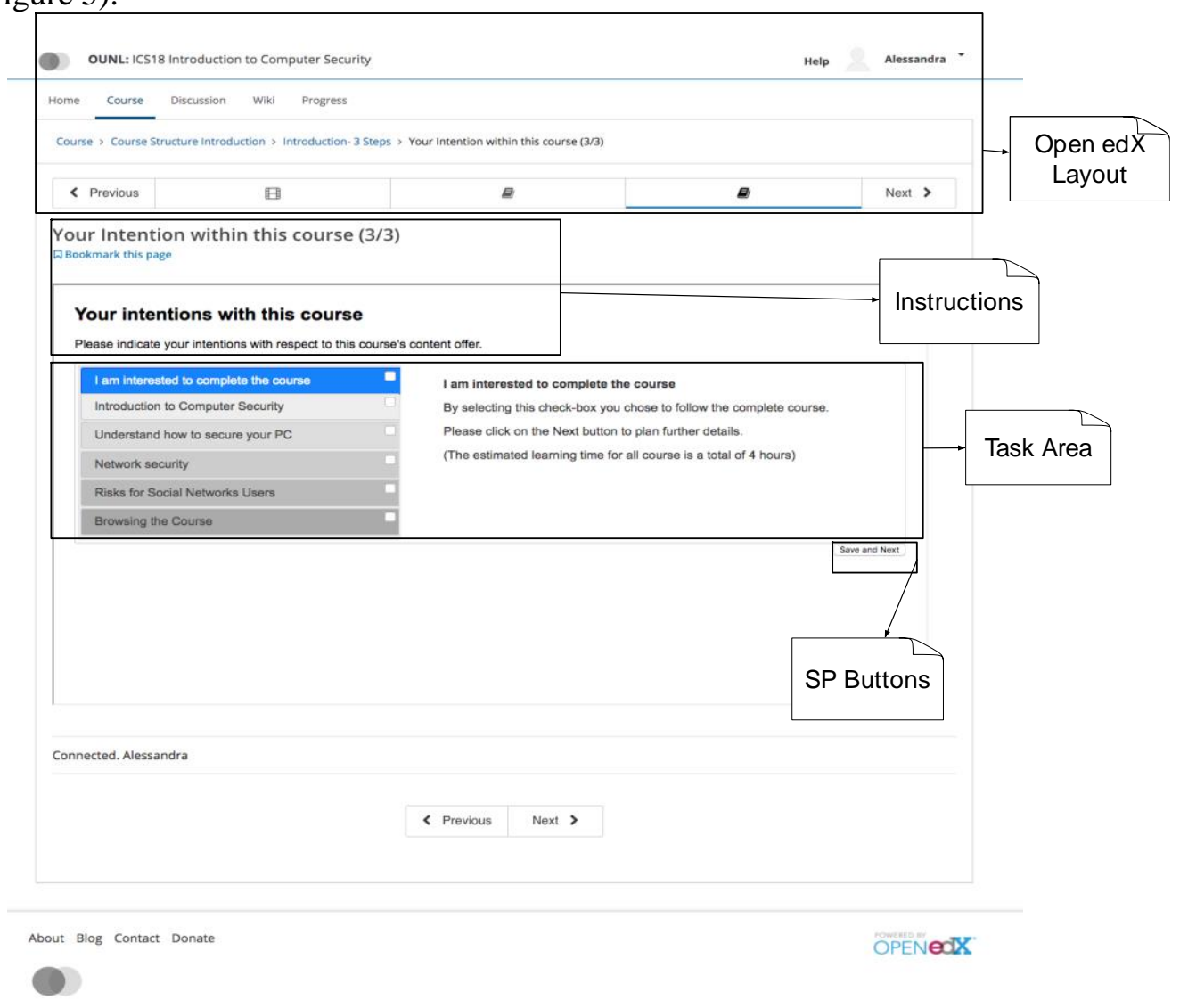

Figure 5. AOI drown on the "intention page", first interface of the SP

An immediate and chromatic way to gain insights on the users visual patterns, performed by the eye of our participants on each interface, are the heat maps. The heat maps 
pag. 60

show, through gradation of colours (blue, green, yellow and red ), the number and length of the fixations; the warmer is the colour of the area/spot, the more time has been spent by the eyes of our participants in that specific spot of the AOI. Here below the heat maps of the SP (Figure 6) are represented.

$1^{\text {st }}$ widget- Intention Page

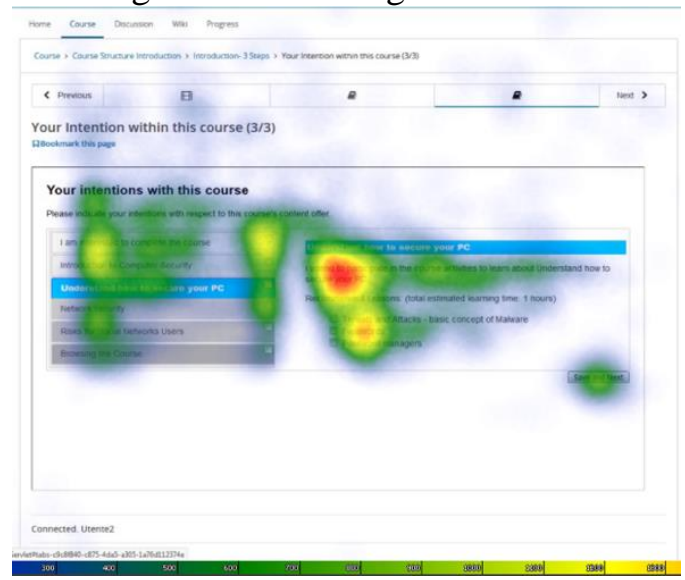

$3^{\text {rd }}$ widget- Memo Page

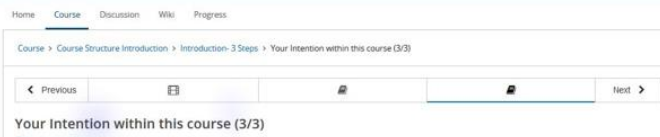

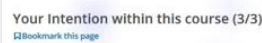
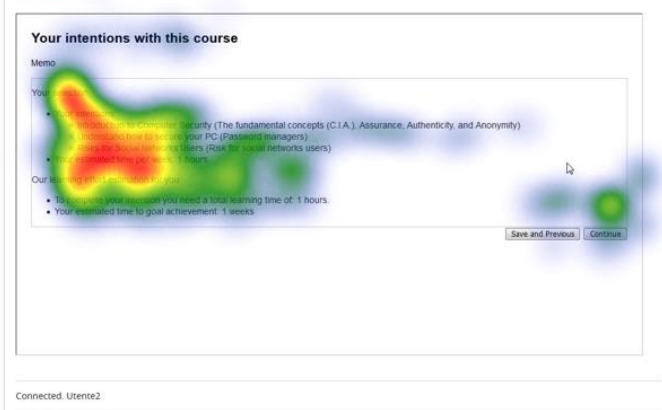

$5^{\text {th }}$ widget- Plan " $b$ " Page
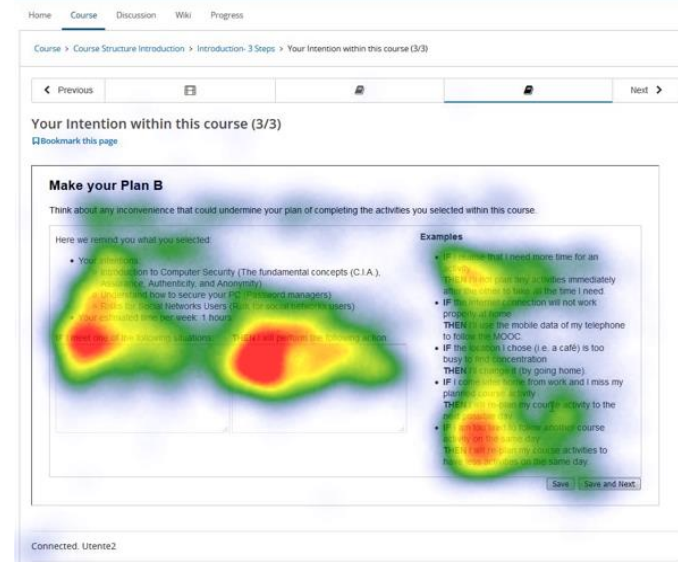

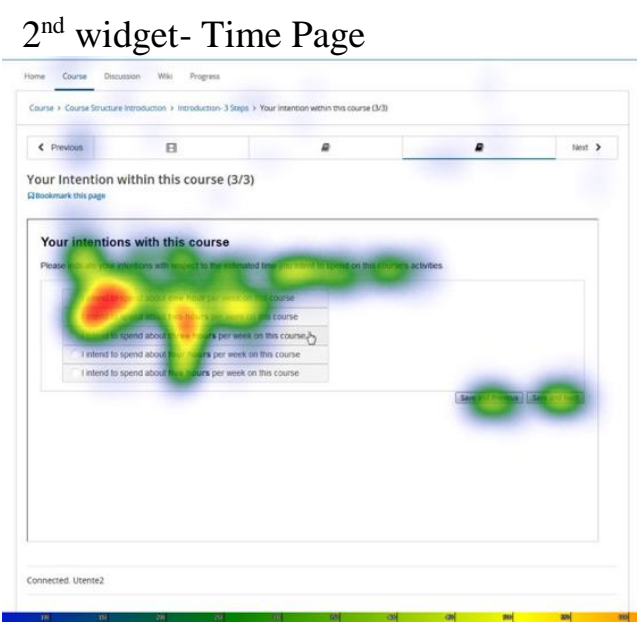

$4^{\text {th }}$ widget- Plan Page

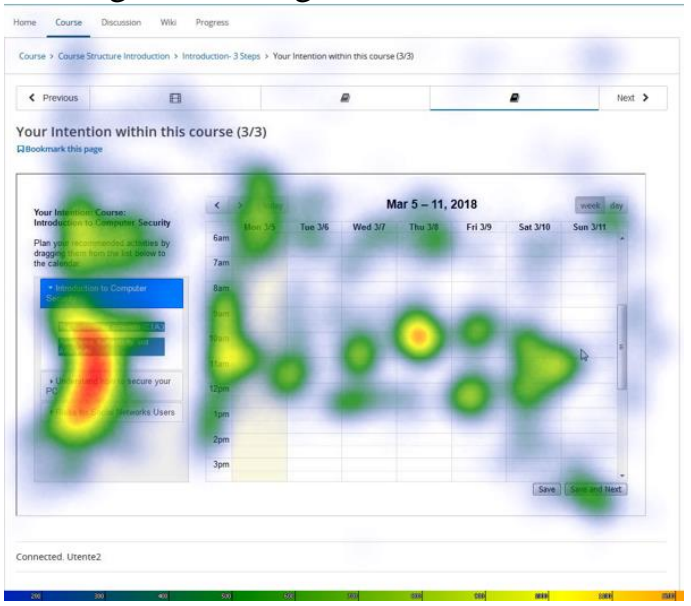

$6^{\text {th }}$ widget- The End Page
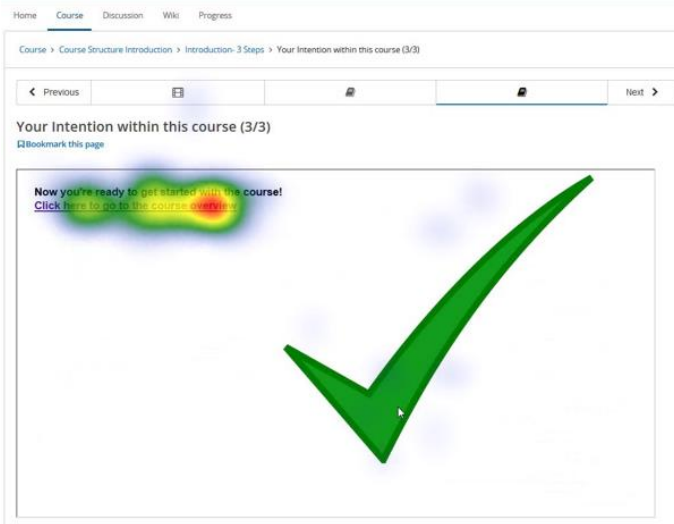

comeneres unenter

Figure 6. Heat Maps of 6 Stimulated Planning Interfaces 
In addition, for each AOI, we extracted the normalized dwell average, the revisits average and the fixation duration average per each of the AOI on each interface with the aim of compare them and gain insights from it.

Starting with the AOI- Open edX Layout, from the data, it is evident that the AOI- Open edX layout did attract participants' attention, mostly on the first interface- the "intention page", yielding a normalized dwell average of 20932.7/ms (Table3).

Table 3. Open edX Layout area

\begin{tabular}{c|l|c|c|c}
\hline No. & Stimulus & $\begin{array}{c}\text { Normalized Dwell } \\
\text { Average } \\
\text { [ms/Coverage] }\end{array}$ & $\begin{array}{c}\text { Revisits } \\
\text { Average }\end{array}$ & $\begin{array}{c}\text { Fixation Duration } \\
\text { Average [ms] }\end{array}$ \\
\hline 1 & Intention Page & 20932.7 & 4 & 202.2 \\
\hline 2 & Time Page & 4531.8 & 0.3 & 135.5 \\
\hline 3 & Memo Page & 0 & 0 & 0 \\
\hline 4 & Plan Page & 12102.7 & 4 & 62.2 \\
\hline 5 & Plan "b" Page & 377.7 & 0 & 26.2 \\
\hline 6 & End Page & 298.7 & 0 & 38.1 \\
\hline
\end{tabular}

While, the average normalized dwell on the first interface is $20932.7 / \mathrm{ms}$ with revisit average of 4 times and a fixation duration average of $202.27 / \mathrm{ms}$. Attention to this specific AOI decreases progressively on the following page and reaches 0 in the "Memo Page", then rises again in the "Plan Page" where the task given is a little more complex. In this page (Plan Page) the normalized dwell average is $12102.7 / \mathrm{ms}$ with a revisit average of 4 times, and an average fixation duration of $62.2 / \mathrm{ms}$. The reason for this visual pattern can be in the low learnability of this page; probably to find orientation, participants started to look at the top of the page where the AOI - Open edX layout is displayed. However, by consulting the heat maps, it is visible that the AOI- Open edX Layout never registered a warm colour, it is always represented in blue, which means that participants have looked at this AOI (fixation) but not for too long.

As far as the AOI- Instructions is concerned, the data represented in Table 4, shows that the instructions captured the attention of users in different ways on each of the SP interfaces.

Table 4. Instruction area of each page/widget of the SP

\begin{tabular}{c|l|c|c|c}
\hline No. & \multicolumn{1}{|c|}{ Stimulus } & $\begin{array}{c}\text { Normalized Dwell } \\
\text { Average } \\
\text { [ms/Coverage] }\end{array}$ & $\begin{array}{c}\text { Revisits } \\
\text { Average }\end{array}$ & $\begin{array}{c}\text { Fixation Duration } \\
\text { Average [ms] }\end{array}$ \\
\hline 1 & Intention Page & 143962.8 & 8.4 & 277.5 \\
\hline 2 & Time Page & 49825.9 & 1.9 & 324.5 \\
\hline 3 & Memo Page & 10746.4 & 0 & 187 \\
\hline 4 & Plan Page & 176759.2 & 5.8 & 306.2 \\
\hline 5 & Plan "b" Page & 283318.8 & 19.3 & 335.4 \\
\hline 5.2 & Plan "b" examples & 460877.7 & 15 & 348 \\
\hline 6 & End Page & 120364.1 & 1.1 & 294.6 \\
\hline
\end{tabular}

More specifically from Table 4, it is possible to deduce that the instructions on the "Memo Page" were not revisited, while the instructions on the "Plan b" page were revisited on average 19.3 times, a bit more than the instructions on the "plan $b$ page" in the "examples" area, which were revisited on average 15 times. Apparently, participants needed to go back to the instructions quite a lot to be able to perform the task.

The "plan b page" presented two instruction areas: on top of the page (labelled in the table 4 "plan b page"), and on the right-middle side of the page ("plan b examples"). These areas yielded the highest average values for all the measures. More specifically the highest 
pag. 62

normalized dwell were recorded by "plan b examples" (460877.7/ms) and "plan b page" (283318.8/ms). As far as the highest level of fixation duration average is concerned, is yielded respectively by the "plan b examples" (348/ms) and the "plan b page" (335.4/ms). While the lowest average values of normalized dwell (10746.4/ ms) and fixation duration $(187 / \mathrm{ms})$ were instead recorded on the "Memo Page". As a consequence, participants did read the instructions on each page, to a different degree in relation to the interface, the amount of text did not stop participants from reading.

The heat maps confirm what the data showed, indeed, among all the interfaces of the SP, the "plan b page" has the warmer colours on the AOI- instructions, and specifically in the examples area (right middle side).

Examining the AOI - task we calculated only the average fixation duration to understand if the task area attracted users' attention. On each interface we drew one AOI task, except for the "Plan page" where it was needed to draw two: Plan page- calendar and plan page-lessons.

Table 5. Task areas on the page/widget

\begin{tabular}{c|l|c}
\hline No. & \multicolumn{1}{|c}{ Stimulus } & $\begin{array}{c}\text { Fixation Duration } \\
\text { Average [ms] }\end{array}$ \\
\hline 1 & Intention Page & 306.8 \\
\hline 2 & Time Page & 387.6 \\
\hline 3 & Memo Page & 324 \\
\hline 4.1 & Plan Page (calendar) & 421.5 \\
\hline 4.2 & Plan Page (lessons) & 352.7 \\
\hline 5 & Plan "b" Page & 329.6 \\
\hline 6 & End Page & 294.6 \\
\hline
\end{tabular}

The data reported in Table 5, show that participants spent more time by looking at the task on the "plan page (calendar)", with a fixation duration average of $421.5 / \mathrm{ms}$, followed by the "time page" of the SP, with a value of $387.6 / \mathrm{ms}$. While, the lowest fixation duration average was on the "end page", with $294.6 / \mathrm{ms}$. A reason for this visual pattern can lie in the fact that the calendar covered a big area of the "plan page", furthermore the execution of the task required to fixate at a specific area of the calendar to plan the lessons. A narrow and specific area on which to focus can be the reason of the high fixation duration on "time page", which presented a selection of options in a specific area of the interface.

Table 6. SP Buttons

\begin{tabular}{c|l|c|c|c}
\hline No. & \multicolumn{1}{|c|}{ Stimulus } & $\begin{array}{c}\text { Normalized Dwell } \\
\text { Average } \\
\text { [ms/Coverage] }\end{array}$ & Revisits Average & $\begin{array}{c}\text { Fixation } \\
\text { Duration Average } \\
\text { [ms] }\end{array}$ \\
\hline 1 & Save and Next & 373528.3 & 3 & 330.9 \\
\hline 2.1 & Save and Next & 146098.3 & 0.6 & 405.1 \\
\hline 2.2 & Save and Previous & 102151.6 & 0.5 & 395.7 \\
\hline 3.1 & Continue & 67249.5 & 0 & 160.6 \\
\hline 3.2 & Save and Previous & 16161.0 & 0 & 60.5 \\
\hline 4.1 & Save and Next & 460771.9 & 1.5 & 310.9 \\
\hline 4.2 & Save & 197748.2 & 0.7 & 221.4 \\
\hline 5.1 & Save and Next & 186743.0 & 1.8 & 375.2 \\
\hline 5.2 & Save & 347979.5 & 1.3 & 218.5 \\
\hline
\end{tabular}

Finally, we wanted to understand if the Open edX buttons "next/previous", positioned at the bottom of our SP were a distractor for the SP buttons. Furthermore, it was in the 
authors interest to understand the most appropriate wording options to be used in the button. On each interface, we adopted different terminologies for SP button, more specifically: save previous, save next, continue, as detailed in the Stimulus column, in table 6.

Based on the results, all participants used the SP buttons correctly without clicking on the "Next" button of Open edX. Furthermore, from the data (Table 6), it can be highlighted that the "Save and Next" as command button gained more revisits compared to the "continue" button, with zero revisits. Apparently, our participants were confused by the option "Save and Next" and needed to look at the area more often before understanding its function.

\subsection{Improvements level (RTA)}

The results of the RTA were used to gain a better understanding of the ET data and the improvements needed (RQ3).

A total of 208 segments of content- and task-related statements (question statements excluded) were collected in the think-aloud interviews. Following [33] guide, we clustered each utterance (segment of the conversation) in six dimensions: (1) layout; (2) comprehension; (3) data entry; (4) feedback; (6) navigation and (7) users' suggestions.

Two independent encoders were trained to code the interview related to the "Plan Page" which constitutes $40 \%$ of the total data. Cohen's Kappa as an indicator of inter-rater agreement was calculated and it was satisfactory $(\kappa=0.49)$ (kappa value between $0.40-$ 0.75 implies fair to good agreement).

To give the reader a better understanding of the coding applied, Table 7 provides the description of each dimension and a statement as example. While Figure 7 represents the amount of participants comments ordered per dimension and interface of the SP.

Table 7.Coding matrix

\begin{tabular}{|c|c|c|}
\hline Dimensions & Description & Sample Statement \\
\hline \multirow[b]{2}{*}{ Layout } & Utterances related only to the visualization & "the layout was nice" \\
\hline & $\begin{array}{l}\text { Utterances related to the necessity (or not) of the } \\
\text { information given }\end{array}$ & "the layout was clear/understandable" \\
\hline \multirow{2}{*}{ Comprehension } & $\begin{array}{l}\text { Utterances related to comprehension of the } \\
\text { instructions given }\end{array}$ & $\begin{array}{c}\text { "by reading the instructions I didn't get } \\
\text { what to do" }\end{array}$ \\
\hline & $\begin{array}{l}\text { Utterances related to the formulation of the } \\
\text { instructions and information given }\end{array}$ & $\begin{array}{l}\text { "I'll formulate the sentence in another } \\
\text { way here" }\end{array}$ \\
\hline Data Entry & $\begin{array}{l}\text { Utterances related to entering information or using } \\
\text { functionalities of the system to complete a task }\end{array}$ & "I manage to drag and drop it" \\
\hline Feedback & $\begin{array}{l}\text { Utterances related to the coherence and consistency } \\
\text { of the feedback given by the system }\end{array}$ & $\begin{array}{l}\text { "I saw on the previous page that I needed } \\
\text { two weeks to complete the course but, in } \\
\text { the calendar, couldn't find the two weeks } \\
\text { to plan" }\end{array}$ \\
\hline Navigation & $\begin{array}{l}\text { Utterances related to the navigation within the } \\
\text { system }\end{array}$ & "I liked it, it was smooth" \\
\hline \multirow{2}{*}{$\begin{array}{c}\text { Users' } \\
\text { suggestions }\end{array}$} & \multirow{2}{*}{ Suggestions from users on how to improve the SP } & "I would not use save but just next' \\
\hline & & "I would put more icons here" \\
\hline
\end{tabular}

Each interface of the SP was subject to comments from the participants. The interfaces with more utterances codified, in descending order, are the "intention page" and the "plan page", with an equal number of comments: 50; followed by the "plan b page", with 43 utterances; "the memo page", which collected 29 codified utterances; "the time page" with 23 codified participants' statements and finally the least amount of comments were related to "the end page" with 13 utterances codified.

As visually represented in Figure 7, below, the dimension more commented and therefore most discussed during the RTA interviews, was "layout", with 71 comments codified, followed by "users' suggestions", with 41 comments coded. Then "Data Entry" 
and "Comprehension" with respectively 39 and 35 utterances clustered. Finally, "feedback with 13 utterances and "Navigation" with nine comments.

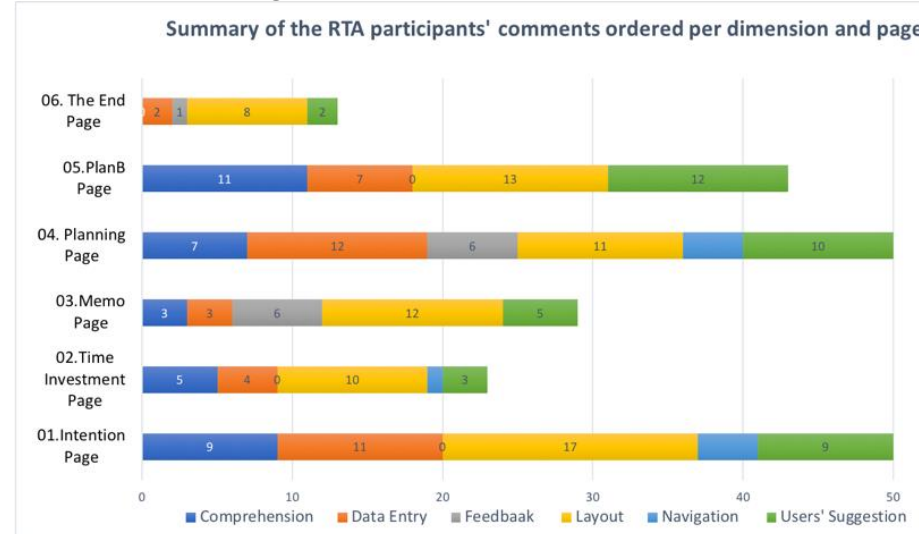

Figure 7. Summary of the RTA participants' utterances ordered per dimension and widget/page of the Stimulated Planning

\section{The participants' voice: comments per widget/page of the SP and dimension}

01. Intention page

In the following, the summary of the participants' comments on the $1^{\text {st }}$ widget/page of the SP, the "intention page", per dimension are reported:

- Layout: the majority of participants found overall "the interface layout not immediate", however the task area position, in the middle, was well perceived. The colour choice instead created confusion in particular the shades of grey chosen. Participants were asked about the Open edX layout and the dashboard and three out of eight declared that they were attracted by it and they justified it by saying that they looked at it because they were studying all the page to understand what to do and it was easy to understand, after some seconds, that the relevant information for the execution of the task was in the centre.

- Comprehension: which is the dimension related to participants' understanding of the instructions given on the page and on the terminology used. Only two people reported understanding the information in a "clear" way, for the rest the information was be reformulated.

- Data Entry: as far as the box selection is concerned, the click on the first box that selects all the options created some confusion; it was also not clear if the presence of the button "save" alone and the "save and next" on the same page. Some of the participants thought that the "save" was there because they needed to save every time they selected an option, "in some programs you have to save every step so I was not sure about that".

- Feedback was not commented

- Navigation: received positive comments, it "was smooth" for all the participants that made a comment related to this dimension.

- Users' suggestions related to this page were several and mainly related to the interface layout, such as: "the interface will be more immediate if you put two options, on one button for all the course and the other that enables users to select which activities to do within the course"; or to insert drop-down menu icon to indicate that indicate. Other suggestions were related to the terminology. The word "intention" made some participants doubtful of what to do and a more direct framing of the sentence was proposed, such as: "what do you plan to do in this course?" instead of "select your intention within the course". Finally, it was suggested to use just one button as SP button with the label "next" or "continue" and eliminate the confusing "save". 
02- Time Page

In the following, the summary of the participants' comments on the $2^{\text {nd }}$ widget/page of the SP, the "time page", per dimension are reported:

- Layout: from the utterances coded the layout used was well perceived by users, even though some of them would prefer less text to be read.

- Comprehension: the terminology used was understood by users and, according to participants, the alternation of normal and, the bold font in the text speeds up the cognitive process.

- Data entry for the task execution worked well, while the term "save" on the SP button created, also on this page, the same misunderstanding reported for the first widget of the SP.

- Feedback: there were no utterances clustered under this dimension.

- Navigation: was well perceived and commented by users.

- Users' suggestions for improving this page were mainly related to the SP buttons, "I would just put previous and next it doesn't matter if you save or not if you click next you assumed it is saved".

\section{3- Memo Page}

In the following, the summary of the participants' comments on the $3^{\text {rd }}$ widget/page of the SP, the "memo page", per dimension are reported:

- Layout of this page was generally well perceived because "it looks like a memo", two participants did not find it very attractive because of its simplicity and amount of text.

- Comprehension, the terminology used was not easy to understand, people "needed to read it twice".

- Data entry was well perceived.

- Feedback: This widget is per se a feedback and as such it was positively commented.

- Users' suggestions provided were related to the layout: "it would be better (to have) a layout that, by looking at it, gives you all the info without the need of reading all".

04- The Plan Page

In the following, the summary of the participants' comments on the $4^{\text {th }}$ widget/page of the SP, the "plan page", per dimension are reported

- Layout: the majority of participants were visually attracted by the calendar, so they liked the layout.

- Comprehension: participants were so much attracted by the calendar that they skipped the instructions on this page. As a consequence, the comprehension was low.

- Data entry worked well. The dragging and dropping mechanism for the lessons plan worked for almost all the users.

- Users' suggestions were related:

- the layout: for some participants it would have been better to insert a progress bar that informs them on the activities already planned and the ones to do;

- feedback and information, participants declared the need of having more guidance in planning the lessons and more explicit info on the lessons' duration.

- Instructions: it was also proposed to use a more direct approach in the instructions given, something like "now we are going to make your schedule". 


\section{5- The Plan "b" Page}

In the following, the summary of the participants' comments on the $5^{\text {th }}$ widget/page of the SP, the "plan b page", per dimension are reported:

- Layout: the amount of text on this page was liked and disliked equally by the participants of our study ("I like that because it fills the screen" and "it was too much text probably").

- Comprehension: the instructions given were clear, also because there were a lot of examples provided. The terminology used collected positive comments too. Even though, some of the participants lacked information on how many "if" and "then" plans needed to be made.

- Data entry worked well for all, but some of the participants declared that the task per se was difficult, "it was difficult to think about plan b situations".

- Navigation and feedback: no utterances were coded under these two dimensions.

- Users' suggestions were mainly related to layout and data entry of the page. It was indeed proposed to insert an info button on the widget that could contain all the examples. In this way, the layout would look more organized. To improve the guidance on how to complete the task, introducing, for instance, a drop-down menu (with option "other" available) for the problems (if) and the solutions (then) from where users could pick the most relevant if and then for them

06- The End Page

It was well perceived in all the dimensions.

\section{Discussion}

In this study, we aimed at assessing SP prototype on three levels: overall usability; HCI and improvements needed.

To perform the study, we used three compatible methods: SUS, ET and RTA and collected positive results. Via the SUS, we answered RQ1 (How usable is the SP system?) reporting an above average score for the SP. Moreover the SQ1 (To which extent is the SP efficient, effective, learnable and satisfy users?) highlighted positive feedback from the SP in terms of efficiency, effectiveness, learnability and users' satisfaction. With the ET, we gained more insights on the visual pattern of users and a more objective understanding on what attracts user attention and focus. Based on ET and RTA data, we could answer RQ2 (On each interface of the SP, do users focus on the areas considered relevant by the designers?).

RQ2 has been addressed on two levels: page level and AOI.

On a page level: The interfaces on which users spent on average more time (average fixation duration) were "Plan page" and "Plan b page". These are the two pages with more text and complex tasks to solve. The users' perception of the complexity of the task on these pages is confirmed by the blink frequency. The "plan b page", in particular, recorded the highest score, meaning that participants needed more cognitive effort to understand how to perform the task (the more difficult the task, the higher the blink frequency). What is instead surprising is that the "time page" even if simple, also scored, on average a high fixation duration, suggesting the need of participants to focus longer on this widget. This can be due to the fact that the "time page' is the second interface that users visualize and it presents a different layout compared to the first, so probably, participants needed some time to get acquainted with it. It is instead not surprising that the "end page" had the lowest values because its layout is very intuitive and does not require focus. Much more cognitive effort was instead needed for the "plan b page", with the higher blink frequency registered. As 
also the RTA confirmed, it was not easy for participants to produce the coping plan, even though they reported that the instructions were clear.

On AOI level: by analysing the AOI on each interface, we managed to respond to the RQ2. The users focused on the area that was most relevant without being distracted by the Open edX layout. Participants read the instructions, even when the text was dense (examples in the plan "b" page), managed to focus on the relevant area and understood how to solve the tasks given. More specifically, the Open edX layout was not an obstacle or generator of confusion in the navigation, instead the average normalized dwell decreased from the first widget on, and none of the participants have used the Open edX buttons to change page. Furthermore, based on the fixation duration and normalized dwell average, participants in our study read the instructions given but they needed to review them several times in the "plan b page" in order to perform the task, suggesting us to reconsider the way in which the instructions were formulated in this widget.

Other suggestions on how to improve the SP prototype (RQ3) come from the users' RTA data analysis. The suggestions were mainly related on the dimensions layout and comprehension and will be considered for the second version of SP tool.

Overall these results exceeded our expectations, on two levels. The quality of data gained by combining three different (but complementary) methods and the positive evaluation of the first SP prototype received.

Compared to previous work, specifically usability studies of game elements applied in MOOCs, this work shows that the combination of three different methods (SUS, ET \& RTA) enables designers to grasp a better understanding on the usability of their system.

\section{Conclusions}

Recognizing the importance of goal achievement for MOOC learners, this study presents a technical solution to enable them follow their intentions within a MOOC scenario. The solution proposed is a game element, that we designed by taking inspiration from (1) the implementation intention theory, from which it takes the structure; and (2) from typical strategy games, from which it gains the mechanism and the logic. This solution is the Stimulated Planning (SP) game element of which this study has presented the architecture and technical implementation details. Furthermore its usability has been investigated, in terms of efficiency, effectiveness, learnability (RQ1 and SQ1), HCI (RQ2) and possible improvements (RQ3).

The results on the one hand show that our participants evaluated the SP prototype as highly usable, on the other enabled us identifying the improvement needed, mainly at level of layout and terminology used. Detecting gaps was one of the reasons why we performed the study, so that we can address and overcome them in the next versions of the SP.

We argue that these results are an important step for the gamification of MOOC, moving the focus from external rewards, mainly in the shape of Points, Badges and Leaderbords (PBL), to a more advanced gamification. By this study the authors, indeed, attempt to demonstrate that gamification can be applied with more complex game elements within MOOCs. Furthermore, it showcases the introduction of a new theory not yet applied in the field: implementation intention to enhance goal achievement, making a step towards the personalization of MOOC and introducing a new way of measuring the MOOC completion rate. Finally, our study proposes a multi-methods approach to be applied in the assessment of a game element prototype that is new in the field.

An important lesson learned and key outcome for MOOC designers and developers, who aim to gamify their MOOCs by applying game-design thinking, is, that learner experience depends to a large extent on a good balance between course content, selected game elements, and usability of these elements in the context of the overall course (and the platform, in which the course is embedded in). While many gamification approaches apply what we call "shallow" gamification, which mainly focuses on the transfer of standard 
learning progress measures into game-like visualizations (in the form of PBL), "deep"/advanced gamification requires a more careful design. Our work has explored the design and test of an advanced game element that implies for its functionalities to embed and balance support for various game soft skills (empowerment, illusion of influence strategic planning), and process/game mechanics (feedback, progress tracking and the replanning).

Our previous work shows that the choice and design of game elements needs to be balanced very thoughtfully to the problem at hand, the course structure, the target audience and the MOOC platform in which the course is embedded [34]. More advanced gamification concerns the conceptualization and creation phase of the game elements, but also to the implementation, which is another crucial step, for the choices to be made. The same game element, indeed, can be perceived as less meaningful for users, when not well implemented. An example for this is the confusion some users experienced in different levels of navigation in our early prototype. While the MOOC platform obviously comes with its own navigation mechanism, our game element had its own navigation logic, which complicated comprehension for some users.

The next step in our research will concern investigating whether a thoughtful integration of meaningful and well-designed game elements into a MOOC can contribute to the learner experience. Therefore, we plan to run a MOOC with the purpose of empirically testing the effects of our game element on learners. Specifically, we aim to understand whether empowering MOOC users in planning their intentions within the course will affect their goal achievement and therefore the MOOC completion rate. Once the effect of the SP have been tested, a summative study will be performed which will include more game elements aiming at developing a sense of community among MOOC users.

\section{References}

[1] A. S. Sunar, N. A. Abdullah, S. White, and H. Davis, "Personalisation in MOOCs: A critical literature review," in Communications in Computer and Information Science, vol. 583, S. Zvacek, M. Restivo, J. Uhomoibhi, and M. Helfert, Eds. Cham: Springer, 2016, pp. 152168. https://doi.org/10.1007/978-3-319-29585-5_9

[2] P. M. Gollwitzer, "Implementation intentions: Strong effects of simple plans.," American Psychologist, vol. 54. pp. 493-503, 1999. https://doi.org/10.1037/0003-066X.54.7.493

[3] P. M. Gollwitzer and P. Sheeran, "Implementation intentions and goal achievement: A metaanalysis of effects and processes," Adv. Exp. Soc. Psychol., vol. 38, pp. 69-119, 2006. https://doi.org/10.1016/S0065-2601(06)38002-1

[4] P. M. . Gollwitzer and G. Oettingen, "Implementation Intentions," in Encyclopedia of Behavioral Medicine, T. J.R., Ed. New York, NY: Springer New York, 2013, pp. 10431048.

[5] T. Martiny-Huenger, M. Bieleke, G. Oettingen, and P. M. Gollwitzer, "From thought to automatic action: Strategic and spontaneous action control by if-then planning," Reflective Impuls. Determ. Hum. Behav., no. September, pp. 69-84, 2016.

[6] M. A. Adriaanse, C. D. W. Vinkers, D. T. D. De Ridder, J. J. Hox, and J. B. F. De Wit, "Do implementation intentions help to eat a healthy diet? A systematic review and meta-analysis of the empirical evidence," Appetite, vol. 56, no. 1, pp. 183-193, Feb. 2011. https://doi.org/10.1016/j.appet.2010.10.012

[7] J. Reich, "Learner Intention Recasts 'Low' MOOC Completion Rates | HarvardX," 2014. [Online]. Available: http://harvardx.harvard.edu/news/learner-intention. [Accessed: 29-Feb2016].

[8] M. A. Henderikx, K. Kreijns, and M. Kalz, "Refining success and dropout in massive open online courses based on the intention-behavior gap," Distance Educ., pp. 1-16, 2017. https://doi.org/10.1080/01587919.2017.1369006

[9] A. Antonaci, R. Klemke, C. M. Stracke, and M. Specht, "Gamification in MOOCs to enhance users' goal achievement," in IEEE Global Engineering Education Conference, 
EDUCON, 2017. https://doi.org/10.1109/EDUCON.2017.7943070

[10] S. Björk and J. Holopainen, Patterns in Game Design. 2005.

[11] A. Abran, A. Khelifi, W. Suryn, and A. Seffah, "Consolidating the ISO usability models," Proc. 11th Int. Softw. Qual. Manag. Conf., pp. 23-25, 2003.

[12] Z. Yang, Y. Zhang, M. Li, and T. Chen, "The Comparison Study of Usability Test Methodology Based on Eye-Tracking Technology," in Man-Machine-Environment System Engineering. MMESE 2017, 2018, pp. 763-772.

[13] H. Jarodzka, K. Holmqvist, and H. Gruber, "Eye tracking in educational science : Theoretical frameworks and research agendas," J. Eye Mov. Res., vol. 10, no. 1, pp. 1-18, Feb. 2017.

[14] J. K. Jacob and K. S. Karn, "Eye tracking in human-computer interaction and usability research," in The Mind's Eyes: Cognitive and Applied Aspects of Eye Movements Research, no. 1905, J. Hyönä, R. Radach, and H. Deubel, Eds. Elsavier, 2003, pp. 573-605.

[15] A. Antonaci, R. Klemke, C. M. Stracke, and M. Specht, "Gamification in MOOCs to enhance users ' goal achievement," in Proceedings of IEEE Global Engineering Education Conference (EDUCON 2017), 25-28 April, Athens Greece, 2017, pp. 1654-1662. https://doi.org/10.1109/EDUCON.2017.7943070

[16] M. Shubina, "Usability evaluation of MOOC platforms," Haaga-HeliaUniversity of Applied Science, 2016.

[17] S. Johansson and I. Frolov, "An Adaptable Usability Checklist for MOOCs : A usability evaluation instrument for Massive Open Online Courses," University UMEA, 2014.

[18] D. Griffin, "A brief history of Gamifiction," The HR Director, 2014. [Online]. Available: https://www.thehrdirector.com/features/gamification/a-brief-history-of-gamification. [Accessed: 07-Jun-2016].

[19] L. Pappano, "The Year of the MOOC," The New York Times, 2012. [Online]. Available: http://www.nytimes.com/2012/11/04/education/edlife/massive-open-online-courses-aremultiplying-at-a-rapid-pace.html?pagewanted=all\&_r=0. [Accessed: 02-Nov-2012].

[20] A. Steinert, I. Buchem, A. Merceron, J. Kreutel, and M. Haesner, “A wearable-enhanced fitness program for older adults, combining fitness trackers and gamification elements: the pilot study fMOOC@Home,”Sport Sci.Health, vol. 14, no. 2, pp. 275-282, Aug. 2018. https://doi.org/10.1007/s11332-017-0424-z

[21] L. Ramírez-Donoso, J. S. Rojas-Riethmuller, M. Pérez-Sanagustín, A. Neyem, and C. Alario-Hoyos, "MyMOOCSpace: A cloud-based mobile system to support effective collaboration in higher education online courses," Comput. Appl. Eng. Educ., vol. 25, no. 6, pp. 910-926, 2017. https://doi.org/10.1002/cae.21843

[22] A. Antonaci, R. Klemke, K. Kreijns, and M. Specht, "Get Gamification of MOOC right! How to Embed the Individual and Social Aspects of MOOCs in Gamification Design," Int. J. Serious Games, vol. 5, no. 3, 2018.

[23] P. M. Gollwitzer, "Implementation intentions," Am. Psychol., vol. 54, no. 7, pp. 493-503, 1999. https://doi.org/10.1037/0003-066X.54.7.493

[24] J. Brooke, "SUS - A quick and dirty usability scale," Usability Eval. Ind., vol. 189, no. 194, pp. 4-7, 1996.

[25] J. R. Lewis and J. Sauro, "The factor structure of the system usability scale," Lect. Notes Comput. Sci. (including Subser. Lect. Notes Artif. Intell. Lect. Notes Bioinformatics), vol. 5619 LNCS, pp. 94-103, 2009. https://doi.org/10.1007/978-3-642-02806-9_12

[26] SMI, BeGaze Manual, 3.7, Augus., no. July. SMI- SensorMotoric Instruments, 2012. https://doi.org/10.1177/001872089203400407

[27] R. A. Virzi, "Refining the Test Phase of Usability Evaluation: How Many Subjects Is Enough?," Hum. Factors J. Hum. Factors Ergon. Soc., vol. 34, no. 4, pp. 457-468, Aug. 1992.

[28] J. Nielsen, J. Nielsen, and T. K. L, "A mathematical model of the finding of usability problems," in PROCEEDINGS OF ACM INTERCHI'93 CONFERENCE, 1993, pp. 24--29.

[29] J. Nielsen, "Why You Only Need to Test with 5 Users," NN/g Nielsen Norman Group, 2000. [Online]. Available: https://www.nngroup.com/articles/why-you-only-need-to-test-with-5users/. [Accessed: 04-Oct-2018].

[30] R. Macefield, "How To Specify the Participant Group Size for Usability Studies: A Practitioner's Guide," 2009.

[31] A. Bojko, Eye tracking the user experience: A practical guide to research. Rosenfeld Media, 2013.

[32] J. Sauro and J. R. Lewis, Quantifyung the User Experience. Practical Statistics for User Research. Elsevier Inc., 2012.

[33] M. Chi, “Quantifying Qualitative Analyses of Verbal Data : A Practical Guide Quantifying 
pag. 70

Qualitative Analyses of Verbal Data : A Practical Guide,” no. September 2012, pp. 37-41, 2009.

[34] A. Antonaci, R. Klemke, K. Kreijns, and M. Specht, "Get Gamification of MOOC right!," Int. J. Serious Games, vol. 5, no. 3, pp. 61-78, Sep. 2018. https://doi.org/10.17083/ijsg.v5i3.255

[35] B. Vangie, "What is Widget? Webopedia Definition." [Online]. Available: https://www.webopedia.com/TERM/W/widget.html. [Accessed: 20-Nov-2018]. 